\title{
Sums of lens spaces bounding rational balls
}

\author{
PAOLO LisCA
}

\begin{abstract}
We classify connected sums of three-dimensional lens spaces which smoothly bound rational homology balls. We use this result to determine the order of each lens space in the group of rational homology 3-spheres up to rational homology cobordisms, and to determine the concordance order of each 2-bridge knot.
\end{abstract}

57M99; 57M25

\section{Introduction}

This paper is a continuation of [7]. Both papers can be viewed as providing partial answers to the question of Andrew Casson [6, Problem 4.5] asking which rational homology 3-spheres bound rationally acyclic 4-manifolds. The main result of [7] characterizes lens spaces which smoothly bound rational homology balls, while here we deal more generally with connected sums of lens spaces. Our proof relies on Donaldson's theorem about intersection forms of closed, smooth and negative 4-manifolds as well as on some technical results from [7].

Given coprime integers $p>q>0$, we denote by $L(p, q)$ the oriented 3-manifold obtained by performing $(-p / q)$-surgery on the unknot in $S^{3}$. Let $q^{\prime}$ be the unique integer satisfying $p>q^{\prime}>0$ and $q q^{\prime} \equiv 1 \bmod p$. By [7, Theorem 1.2] the lens space $L(p, q)$ smoothly bounds a rational homology ball if and only if

(1) $p=m^{2}$ for some $m \in \mathbb{N}$, and

(2) $q, p-q$ or $q^{\prime}$ is of one of the following types:

(a) $m k \pm 1$ with $m>k>0$ and $(m, k)=1$;

(b) $d(m \pm 1)$, where $d>1$ divides $2 m \mp 1$;

(c) $d(m \pm 1)$, where $d>1$ is odd and divides $m \pm 1$.

Let $\mathcal{R} \subset \mathbb{Q}$ denote the set - described above - of rational numbers $p / q>1$ such that the lens space $L(p, q)$ smoothly bounds a rational homology ball, and define

$$
\mathcal{F}_{n}:=\left\{\frac{m^{2} n}{m n k+1} \mid m>k>0,(m, k)=1\right\} \subset \mathbb{Q}, \quad n \geq 2 .
$$


Theorem 1.1 Let $Y$ be an oriented 3-manifold homeomorphic to a connected sum of three-dimensional lens spaces. Then, $Y$ smoothly bounds a rational homology ball if and only if $Y$ is orientation-preserving homeomorphic to a connected sum in which each summand is (possibly orientation-reversing) homeomorphic to one of the following oriented 3-manifolds:

(1) $L(p, q), p / q \in \mathcal{R}$;

(2) $L(p, q) \# L(p, p-q), p / q>1$;

(3) $L\left(p_{1}, q_{1}\right) \# L\left(p_{2}, q_{2}\right), p_{i} / q_{i} \in \mathcal{F}_{2}, i=1,2$;

(4) $L(p, q) \# L(n, n-1), p / q \in \mathcal{F}_{n}$ for some $n \geq 2$;

(5) $L\left(p_{1}, q_{1}\right) \# L\left(p_{2}, p_{2}-q_{2}\right), p_{i} / q_{i} \in \mathcal{F}_{n}, i=1,2$, for some $n \geq 2$.

Let $\Theta_{\mathbb{Q}}^{3}$ be the group of rational homology 3-spheres up to rational homology cobordism. Using Theorem 1.1 we can determine the order of each lens space viewed as an element of the group $\Theta_{\mathbb{Q}}^{3}$ of rational homology 3-spheres up to rational homology cobordism. Define

$$
\mathcal{S}:=\left\{p / q \mid p>q>0,(p, q)=1 \text { and } p=q+q^{\prime}\right\} \subset \mathbb{Q} .
$$

Corollary 1.2 The order of the lens space $L(p, q)$ in $\Theta_{\mathbb{Q}}^{3}$ is:

- 1 if and only if $p / q \in \mathcal{R}$,

- 2 if and only if $p / q \in(\mathcal{S} \backslash \mathcal{R}) \cup \mathcal{F}_{2}$,

- $\infty$ if and only if $p / q \notin \mathcal{R} \cup \mathcal{S} \cup \mathcal{F}_{2}$.

Since the sets $\mathcal{R}, \mathcal{S}$ and $\mathcal{F}_{2}$ have elementary, explicit definitions, Corollary 1.2 reduces the determination of the order of each lens space in $\Theta_{\mathbb{Q}}^{3}$ to an elementary calculation.

It is a well-known fact that the lens space $L(p, q)$ can be viewed as the 2-fold cover of $S^{3}$ branched along the 2-bridge link $K(p, q)$ (see Section 2 for the definition of $K(p, q))$. As a consequence, Theorem 1.1 gives information on the relation of link concordance amongst 2-bridge links $K(p, q) \subset S^{3}$. Here we content ourselves with deriving a corollary of Theorem 1.1 in the knot case (that is, when $p$ is odd), leaving the case of links to the interested reader. Recall that the concordance order of a knot in $S^{3}$ is the order of its class in the smooth knot concordance group $\mathcal{C}_{1}$. The survey paper of Livingston [8] describes what was known about the group $\mathcal{C}_{1}$ until 2004. More recent papers contain results on knot concordance obtained via Ozsváth-Szabó's Heegaard Floer homology, Grigsby, Ruberman and Strle [4], Jabuka and Naik [5] and Manolescu and Owens [9]. In spite of all the efforts made so far, the structure of the group $\mathcal{C}_{1}$ remains quite mysterious. For instance, the basic question asking whether $\mathcal{C}_{1}$ contains 
nontrivial elements of finite order different from two is still wide open. Restricting to 2-bridge knots, results from [4;5] determine the corresponding concordance orders in finitely many cases.

Corollary 1.3 Let $p>q>0$ with $p$ odd. Then, the 2-bridge knot $K(p, q)$ has concordance order:

- 1 if and only if $p / q \in \mathcal{R}$,

- 2 if and only if $p / q \in \mathcal{S} \backslash \mathcal{R}$,

- $\infty$ if and only if $p / q \notin \mathcal{R} \cup \mathcal{S}$.

Moreover, $K(p, q)$ is simultaneously smoothly slice and amphicheiral if and only if

$$
(p, q)=\left(\left(2 k^{2}+2 k+1\right)^{2}, 2(2 k+1)\left(k^{2}+k+1\right)\right)
$$

for some $k \geq 1$.

Corollary 1.3 corroborates [7, Conjecture 9.4], stating that each knot of order two is concordant to a negative amphicheiral knot. In fact, since $K(p, q)$ is isotopic to $K\left(p, q^{\prime}\right)$ while $K(p, p-q)$ is isotopic to the mirror image of $K(p, q)$, by Corollary 1.3 each 2 -bridge knot of order two is amphicheiral ${ }^{1}$.

The paper is organized as follows. In Section 2 we establish Corollaries 1.2 and 1.3 assuming Theorem 1.1. In Section 3 we prove that the manifolds (1)-(5) of Theorem 1.1 bound rational homology balls. Due to previous results, the only cases which need explicit treatment are (3)-(5). In these cases we show by ribbon moves that each knot bounds a ribbon surface $\Sigma$ with $\chi(\Sigma)=1$. The rational ball is then the 2 -fold cover of $B^{4}$ branched along $\Sigma$ with pushed-in interior. Section 4 is the technical core of the paper. We recall some results from [7] concering embeddings of certain intersection lattices into the standard diagonal lattice, and we prove a few results with a similar flavor. In Section 5 we use the results of Section 4 and Donaldson's theorem on the intersection form of smooth 4-manifolds to finish the proof of Theorem 1.1. Apart from technical issues, the strategy of the proof is very simple. If a connected sum of lens spaces $Y=L_{1} \# \cdots \# L_{h}$ bounds a rational ball $W$ one can construct a closed, negative definite smooth 4-manifold of the form $P_{Y} \cup-W$, where $P_{Y}$ is a certain plumbing canonically associated with $Y$. Donaldson's theorem gives an orthogonal embedding of the intersection lattice of $P_{Y}$ into the standard diagonal intersection lattice. A similar conclusion holds for the intersection lattice of $P_{-Y}$, where $-Y$

\footnotetext{
${ }^{1}$ Since 2-bridge knots are reversible, Siebenmann [13], for such knots the notions of negative and positive amphicheirality coincide.
} 
denotes the 3-manifold $Y$ with reversed orientation. The rest of the proof consists of applying the results of Section 4 to deduce from the existence of such embeddings that $Y$ must be of the form stated in Theorem 1.1.

Acknowledgements I wish to thank Stanislav Jabuka for raising the question which directed me towards Theorem 1.1, and the anonymous referee for several helpful suggestions to improve the quality of the exposition.

\section{Proof of Corollaries 1.2 and 1.3}

In this section we prove Corollaries 1.2 and 1.3 assuming Theorem 1.1.

Proof of Corollary 1.2 By [7, Theorem 1.2] the lens space $L(p, q)$ has order 1 in $\Theta_{\mathbb{Q}}^{3}$, that is, it represents the trivial class, if and only if $p / q \in \mathcal{R}$.

If $L(p, q) \# L(p, q)$ bounds a rational ball, a quick inspection of Theorem 1.1 shows that one of the following must hold:

$$
\text { (1) } p / q \in \mathcal{R}, \quad \text { (2) } p=q+q^{\prime}, \quad \text { or (3) } p / q \in \mathcal{F}_{2} \text {. }
$$

On the other hand, if $L(p, q)$ has order 2 in $\Theta_{\mathbb{Q}}^{3}$ then (1) does not hold. Hence, by the definition of $\mathcal{S}$ we have $p / q \in(\mathcal{S} \backslash \mathcal{R}) \cup \mathcal{F}_{2}$.

Conversely, suppose $p / q \in(\mathcal{S} \backslash \mathcal{R}) \cup \mathcal{F}_{2}$. If $p / q \in \mathcal{S}$ then $q^{\prime}=p-q$, therefore

$$
L(p, q) \# L(p, q)=L(p, q) \# L\left(p, q^{\prime}\right)=L(p, q \# L(p, p-q)=L(p, q) \#-L(p, q)
$$

bounds a rational ball, while $L(p, q)$ does not bound such a ball because $p / q \notin \mathcal{R}$. Therefore $L(p, q)$ has order 2 in $\Theta_{\mathbb{Q}}^{3}$. If $p / q \in \mathcal{F}_{2}$, by Theorem 1.1(3) the connected sum $L(p, q) \# L(p, q)$ bounds a rational ball.

To finish the proof it suffices to show that if $p / q \notin \mathcal{R} \cup \mathcal{S} \cup \mathcal{F}_{2}$ then $L(p, q)$ has infinite order in $\Theta_{\mathbb{Q}}^{3}$. But Theorem 1.1 immediately implies that if $L(p, q)$ has finite order then it has either order 1 or 2 , and therefore by what we have already proved $p / q \in \mathcal{R} \cup \mathcal{S} \cup \mathcal{F}_{2}$.

Before proving Corollary 1.3 we need to recall the definitions of the links $K(p, q)$ and the set $\mathcal{R}$. A link in $S^{3}$ is called 2-bridge if it can be isotoped so it has exactly two local maxima with respect to a standard height function. Figure 1 represents the 2-bridge link $\mathcal{L}\left(c_{1}, \ldots, c_{n}\right)$, where $c_{i} \in \mathbb{Z}, i=1, \ldots, n$. Given coprime integers 

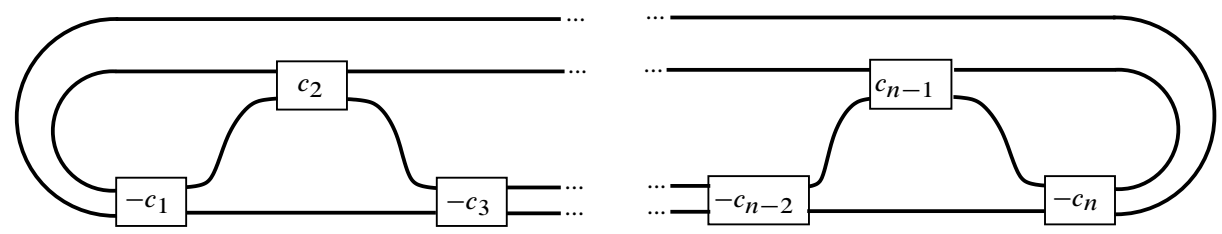

$(n$ odd $)$
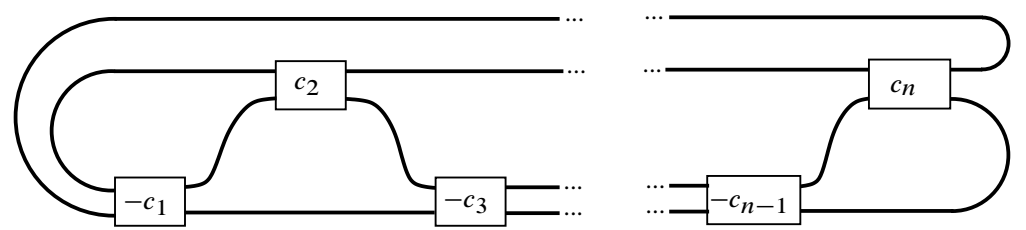

( $n$ even)

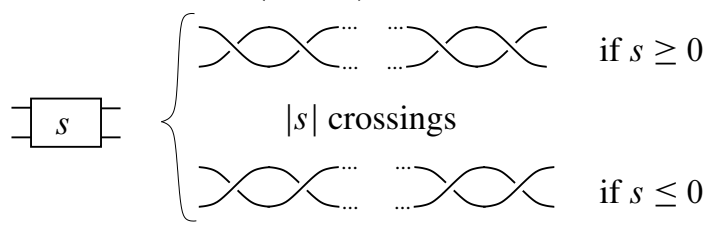

Figure 1: The 2-bridge link $\mathcal{L}\left(c_{1}, \ldots, c_{n}\right)$

$p>q>0$, we can always write

$$
p / q=\left[c_{1}, \ldots, c_{n}\right]^{+}:=c_{1}+\frac{1}{c_{2}+\frac{1}{\ddots \cdot+\frac{1}{c_{n}}}}, \quad \text { with } c_{i}>0, i=1, \ldots, n .
$$

The 2-bridge link $K(p, q)$ is, by definition, $\mathcal{L}\left(c_{1}, \ldots, c_{n}\right)$. When $p$ is even, $K(p, q)$ is a 2-component link, when $p$ is odd $K(p, q)$ is a knot. It is well-known (see Burde and Zieschang [1, Chapter 12]) that $K(p, q)$ and $K(\bar{p}, \bar{q})$ are isotopic if and only if $p=\bar{p}$ and either $q=\bar{q}$ or $q \bar{q} \equiv 1(\bmod p)$, and that every 2-bridge link is isotopic to some $K(p, q)$. Moreover, $K(p, p-q)$ is isotopic to the mirror image of $K(p, q)$.

Proof of Corollary 1.3 If $K(p, q)$ has finite order in $\mathcal{C}_{1}$ then $L(p, q)$ has finite order in $\Theta_{\mathbb{Q}}^{3}$. Therefore by Corollary 1.2 we have $p / q \in \mathcal{R} \cup \mathcal{S} \cup \mathcal{F}_{2}$. If $p / q \in \mathcal{F}_{2}$ then $p$ would be even, while in our case $p$ is odd because we are assuming that $K(p, q)$ is a knot. Hence, $p / q \in \mathcal{R} \cup \mathcal{S}$. Moreover, $K(p, q)$ is smoothly slice if and only if $p / q \in \mathcal{R}$ by [7, Corollary 1.3], while if $p / q \in \mathcal{S}$ then $q^{\prime}=p-q$ and therefore 


$$
\begin{aligned}
K(p, q)= & K\left(p, q^{\prime}\right)=K(p, p-q), \text { so } \\
& K(p, q) \# K(p, q)=K(p, q \# K(p, p-q)=K(p, q) \#-K(p, q)
\end{aligned}
$$

is smoothly slice. This shows that $K(p, q)$ has finite order in $\mathcal{C}_{1}$ if and only if either (i) $p / q \in \mathcal{R}$ and $K(p, q)$ has order 1 or (ii) $p / q \in \mathcal{S} \backslash \mathcal{R}$ and $K(p, q)$ has order 2. This concludes the proof of the part of the statement about concordance orders.

For the last statement, recall that $K(p, q)$ is amphicheiral if and only if $q+q^{\prime}=p$. If

$$
(p, q)=\left(\left(2 k^{2}+2 k+1\right)^{2}, 2(2 k+1)\left(k^{2}+k+1\right)\right)
$$

then, setting $m=2 k^{2}+2 k+1$ and $d=2 k+1$ we have $(p, q)=\left(m^{2}, d(m+1)\right)$ and $d$ divides $2 m-1=d^{2}$. Therefore $p / q \in \mathcal{R}$. Moreover,

$$
p(p-q) \equiv-q^{2}=-d^{2}(m+1)^{2} \equiv(1-2 m)(1+2 m) \equiv 1 \bmod m^{2},
$$

hence $q^{\prime}=p-q$. This shows that if $(p, q)$ is of the stated form then $K(p, q)$ is smoothly slice and amphicheiral.

Conversely, suppose that $K(p, q)$ is smoothly slice and amphicheiral, that is $p / q \in \mathcal{R}$ and $q^{\prime}=p-q$. Since $K(p, p-q)=K(p, q)$, we may assume without loss that $q$ is of the form (a), (b) or (c) from the definition of $\mathcal{R}$. Case (a) is impossible because it is easy to check that $q^{\prime}=m(m-k) \pm 1$, hence $q+q^{\prime}=m^{2} \pm 2 \neq p$. In Case (b), if $q=d(m-1)$ then $q^{\prime}=h(m-1)$, where $h$ is defined by $d h=2 m+1$. Then, $q+q^{\prime}=(d+h)(m-1)=m^{2}=p$ is impossible. If $q=d(m+1)$ we have $q^{\prime}=m^{2}-h(m+1)$, with $d h=2 m-1$. Thus $q+q^{\prime}=p$ if and only if $d=h$, which implies $m=\left(d^{2}+1\right) / 2$, so $(p, q)$ is of the form $\left(\left(d^{2}+1\right)^{2} / 4, d\left(d^{2}+3\right) / 2\right)$. Observing that $d>1$ is odd and setting $d=2 k+1$ one obtains the statement of the lemma. In Case (c), if $q=d(m-1)$ then $q^{\prime}=h(2 m+1)$, with $d h=m+1$, and the equality $q+q^{\prime}=p$ gives a contradiction as in Case (b). If $q=d(m+1)$ then $q^{\prime}=m^{2}-h(2 m-1)$, and the condition $q+q^{\prime}=p$ implies $m=\left(d^{2}+1\right) / 2$ as before, to the effect that $(p, q)$ must be of the stated form.

\section{Proof of Theorem 1.1: first half}

The purpose of this section is to prove the first half of Theorem 1.1, that is, the following result.

Proposition 3.1 Each of the manifolds (1)-(5) listed in Theorem 1.1 bounds a rational homology ball. 
Remark 3.2 According to Siebenmann [13], Casson, Gordon and Conway showed that every knot of the form $K(p, q)$ with $p / q \in \mathcal{R}$ is ribbon. This implies Case (1) of Proposition 3.1 by a standard argument (see Lemma 3.6 or [7] for complete details) Also, in Case (a) of $\mathcal{R}$ Casson and Harer [2] showed that $L(p, q)$ bounds a contractible 4-manifold of Mazur-type.

We start with some preparation. For any integer $t \geq 0$ we will use the notation

$$
\left(\ldots, 2^{[t]}, \ldots\right):=(\ldots, \overbrace{2, \ldots, 2}^{t}, \ldots) .
$$

For example,

$$
\left(3,2^{[3]}, 4\right)=(3,2,2,2,4) .
$$

Recall (see eg Orlik and Wagreich [10, Appendix]) that if $p / q>1$ then

$$
\frac{p}{q}=\left[a_{1}, a_{2}, \ldots, a_{h-1}, a_{h}\right]^{-}:=a_{1}-\frac{1}{a_{2}-\frac{1}{\ddots \cdot-\frac{1}{a_{h}}}}
$$

for some $a_{i} \geq 2, i=0, \ldots, h$, and

$$
\frac{p}{q^{\prime}}=\left[a_{h}, a_{h-1}, \ldots, a_{2}, a_{1}\right]^{-},
$$

where $p>q^{\prime}>0$ and $q q^{\prime} \equiv 1(\bmod p)$. Let $a_{1}, \ldots, a_{2 d}$ be positive integers and $d \geq 2$. Then, the following identity holds (Popescu-Pampu [11, Proposition 2.3]):

(3-2) $\left[a_{1}, \ldots, a_{2 d}\right]^{+}=\left[a_{1}+1,2^{\left[a_{2}-1\right]}, a_{3}+2,2^{\left[a_{4}-1\right]}, \ldots, a_{2 d-1}+2,2^{\left[a_{2 d}-1\right]}\right]^{-}$

We define the reverse of a string $\left(a_{1}, \ldots, a_{k}\right)$ to be $\left(a_{k}, \ldots, a_{1}\right)$, and the negative (respectively positive) fraction of $\left(a_{1}, \ldots, a_{k}\right)$ to be the rational number $\left[a_{1}, \ldots, a_{k}\right]^{-}$ (respectively $\left[a_{1}, \ldots, a_{k}\right]^{+}$).

Lemma 3.3 Suppose that a string $\mathcal{S}$ is obtained from $(2, n+1,2), n \geq 2$, by a finite sequence of operations each of which is of one of the following types:

(1) $\left(n_{1}, n_{2}, \ldots, n_{b-1}, n_{b}\right) \mapsto\left(2, n_{1}, n_{2}, \ldots, n_{b-1}, n_{b}+1\right)$,

(2) $\left(n_{1}, n_{2}, \ldots, n_{b-1}, n_{b}\right) \mapsto\left(n_{1}+1, n_{2}, \ldots, n_{b-1}, n_{b}, 2\right)$.

Then, the negative fraction of $\mathcal{S}$ is of the form

$$
\frac{m^{2} n}{m n k+1}, \quad m>k>0, \quad(m, k)=1 .
$$

Algebraic 83 Geometric Topology, Volume 7 (2007) 
Moreover, the negative fraction of either $\mathcal{S}$ or the reverse of $\mathcal{S}$ is equal to

$$
\left[c_{s}, c_{s-1}, \ldots, c_{2}, c_{1}, 1, n-1, c_{1}+1, c_{2}, \ldots, c_{s}\right]^{+}
$$

for some integers $c_{1}, \ldots, c_{s} \geq 1$, where for $s=1$ the above formula is to be interpreted as $\left[c_{1}, 1, n-1, c_{1}+1\right]^{+}$.

Proof The negative fraction of $(2, n+1,2)$ is $4 n /(2 n+1)$, which is of the form $m^{2} n /(m n k+1)$ for $m=2$ and $k=1$. Therefore it suffices to show that if the negative fraction of a string $\mathcal{S}$ is of the form $m^{2} n /(m n k+1)$, then the negative fractions of the strings obtained from $\mathcal{S}$ by applying the operations (1) and (2) are of the same form. Let

$$
\begin{array}{r}
p:=m^{2} n, \quad q:=m n k+1, \quad q^{\prime}:=m n(m-k)+1, \\
t:=n k(m-k)+1, \quad q^{\prime \prime}:=2 q-n k^{2}=q+t .
\end{array}
$$

Then, we have $0<q^{\prime}<p,\left(p, q^{\prime}\right)=1$,

$$
q q^{\prime}=1+t p \quad \text { and } \quad q^{\prime \prime} q^{\prime}=1+t\left(p+q^{\prime}\right) .
$$

Therefore if $p / q=\left[a_{1}, \ldots, a_{h}\right]^{-}$, by Equation (3-1)

$$
\left[a_{h}+1, a_{h-1}, \ldots, a_{2}, a_{1}\right]^{-}=\frac{p+q^{\prime}}{q^{\prime}} .
$$

Since $0<q^{\prime \prime}=q+t<q+q^{\prime}<p+q^{\prime}$ and $\left(q^{\prime \prime}, p+q^{\prime}\right)=1$, we have

$$
\left[a_{1}, a_{2}, \ldots, a_{h-1}, a_{h}+1\right]^{-}=\frac{p+q^{\prime}}{q^{\prime \prime}}
$$

and therefore

$$
\left[2, a_{1}, a_{2}, \ldots, a_{h-1}, a_{h}+1\right]^{-}=2-\frac{q^{\prime \prime}}{p+q^{\prime}}=\frac{(2 m-k)^{2} n}{(2 m-k) n m+1},
$$

with $2 m-k>m$ and $(2 m-k, m)=1$. Applying Equation (3-6) to

$$
\left[a_{h}, \ldots, a_{1}\right]^{-}=\frac{m^{2} n}{m n(m-k)+1}
$$

gives

$$
\left[2, a_{h}, \ldots, a_{1}+1\right]^{-}=\frac{(m+k)^{2} n}{(m+k) n m+1} .
$$

By Equation (3-1) and the formula for $q^{\prime}$ in Equation (3-4) we have

$$
\left[a_{1}+1, a_{2}, \ldots, a_{h-1}, a_{h}, 2\right]^{-}=\frac{(m+k)^{2} n}{(m+k) n k+1} .
$$


This proves the first part of the lemma. To prove the second part, observe that a straightforward induction using the definition shows that either $\mathcal{S}$ or its reverse is of one of the forms:

$\left(c_{s}+1,2^{\left[c_{s-1}-1\right]}, c_{s-2}+2, \ldots, c_{3}+2,2^{\left[c_{2}-1\right]}, c_{1}+2, n+1,2^{\left[c_{1}\right]}, c_{2}+2, \ldots, c_{s-1}+2,2^{\left[c_{s}-1\right]}\right)$

or

$\left(c_{1}+1, n+1,2^{\left[c_{1}\right]}\right)$.

for some positive integers $c_{1}, \ldots, c_{s}$. Applying Formula (3-2) we obtain Expansion (3-3).

Lemma 3.4 Let $m>k>0$ and $n \geq 1$ be integers, with $(m, k)=1$. Then,

$$
\frac{m^{2} n}{m n k+1}=\left[a_{1}, \ldots, a_{h}\right]^{-}
$$

where the string $\left(a_{1}, \ldots, a_{h}\right)$ is obtained from $(2, n+1,2)$ by a sequence of operations of types (1) or (2) as in Lemma 3.3.

Proof We argue by induction on $m \geq 2$. For $m=2$ we have $k=1$ and

$$
\frac{4 n}{2 n+1}=[2, n+1,2]^{-} \text {. }
$$

Now assume $m>2$ and the conclusion of the lemma for any fraction

$$
\frac{p^{2} n}{p n l+1}, \quad p>l>0, \quad(p, l)=1,
$$

with $p<m$. If $m=2 k$ then $m=2$, therefore we have either $m>2 k$ or $m<2 k$. Since

$$
(m n k+1)(m n(m-k)+1) \equiv 1 \quad\left(\bmod m^{2} n\right),
$$

by Equation (3-1)

$$
\frac{m^{2} n}{m n k+1}=\left[a_{1}, \ldots, a_{h}\right]^{-} \Longleftrightarrow \frac{m^{2} n}{m n(m-k)+1}=\left[a_{h}, \ldots, a_{1}\right]^{-} .
$$

Since string reversal turns the operations (1) and (2) of Lemma 3.3 into each other, up to replacing $k$ with $m-k$ we may assume $m>2 k$ without losing generality. Setting $p:=m-k$ and $l:=k$ we have $m>p, p>l>0$ and $(p, l)=1$. By the induction hypothesis

$$
\frac{p^{2} n}{p n l+1}=\left[b_{1}, \ldots, b_{q}\right]^{-}
$$

Algebraic $8 \mathcal{G}$ Geometric Topology, Volume 7 (2007) 
where $\left(b_{1}, \ldots, b_{q}\right)$ is obtained from $(2, n+1,2)$ by a sequence of operations of types (1) or (2) as in Lemma 3.3. Then, by Equation (3-7) we have

$$
\left(a_{1}, \ldots, a_{h}\right)=\left(b_{1}+1, \ldots, b_{q}, 2\right) .
$$

This concludes the proof.

Lemma 3.5 Let $p / q \in \mathcal{F}_{n}, n \geq 2$. Then, there exists a ribbon move which turns the link $K(p, q)$ into a split link given by the union of $K(n, 1)$ and an unknot.

Proof By Lemmas 3.3 and 3.4, up to isotopy we have the equality

$$
K\left(m^{2} n, m n k+1\right)=\mathcal{L}\left(c_{s}, \ldots, c_{1}, 1, n-1, c_{1}+1, c_{2}, \ldots, c_{s}\right)
$$

for some integers $c_{1}, \ldots, c_{s} \geq 1$. The rest of the proof consists of Figure 2 .

The following lemma can be extracted from [7, Proof of Theorem 1.2]. We include the proof for the reader's convenience.

Lemma 3.6 Let $Y$ be an oriented rational homology 3-sphere given as the 2-fold cover of $S^{3}$ branched along a link $L$ which bounds a ribbon surface $\Sigma \uparrow S^{3}$ with $\chi(\Sigma)=1$. Then, $Y$ smoothly bounds a rational homology 4-ball.

Proof The 4-manifold $W$ given as the 2-fold cover of the 4-ball $B^{4}$ branched along a pushed-in copy of $\Sigma$ smoothly bounds $Y$. Moreover, $W$ has a handle decomposition with only 0-, 1- and 2-handles (see eg Casson and Harer [2, lemma at pages 30-31]). Therefore, from

$$
b_{0}(W)-b_{1}(W)+b_{2}(W)=\chi(W)=2 \chi\left(B^{4}\right)-\chi(\Sigma)=1
$$

we deduce $b_{1}(W)=b_{2}(W)$. On the other hand, since $b_{1}(Y)=b_{1}(\partial W)=0$ and $H_{1}(W, \partial W ; \mathbb{Q}) \cong H^{3}(W ; \mathbb{Q})=0$, the homology exact sequence of the pair $(W, \partial W)$ gives $b_{1}(W)=0$, so it follows that $H_{*}(W ; \mathbb{Q}) \cong H_{*}\left(B^{4} ; \mathbb{Q}\right)$.

Proof of Proposition 3.1 As explained in Remark 3.2, Case (1) of the proposition follows from the results of [13]. In Case (2) the result is well-known. To treat Cases (3)(5) we apply Lemma 3.6. First observe that, as shown in Figure 3, there is a connected sum $K(n, 1) \# K(n, n-1)$ which can be turned into an unlink of 2 components by a ribbon move. On the other hand, applying Lemma 3.5 we see that if $p_{i} / q_{i} \in \mathcal{F}_{2}$ then there is a connected sum $K\left(p_{1}, q_{1}\right) \# K\left(p_{2}, q_{2}\right)$ which can be turned by 2 ribbon moves into the connected sum $K(2,1) \# K(2,1)$ of Figure 3 (for $n=2$ ) plus a 2-component unlink. Therefore, a connected sum $K\left(p_{1}, q_{1}\right) \# K\left(p_{2}, q_{2}\right)$ with $p_{i} / q_{i} \in \mathcal{F}_{2}$ can be 

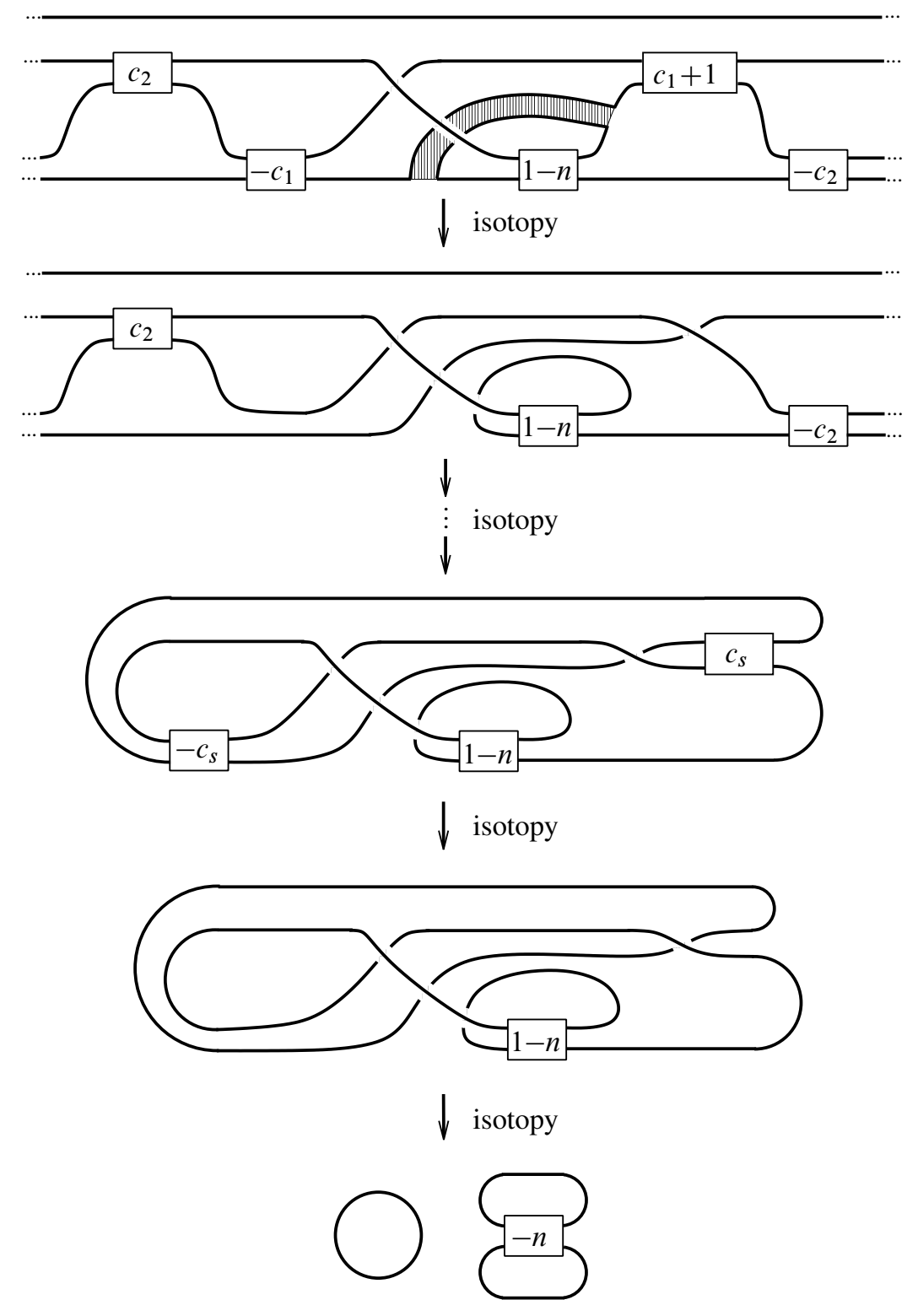

Figure 2: Ribbon move on $K\left(m^{2} n, m n k+1\right)$ and isotopies

turned into a 4-component unlink by 3 ribbon moves. So we conclude that such a link bounds a ribbon surface $\Sigma$ with $\chi(\Sigma)=4-3=1$. The same argument applies to a connected sum $K\left(p_{1}, q_{1}\right) \# K\left(p_{2}, p_{2}-q_{2}\right)$ with $p_{i} / q_{i} \in \mathcal{F}_{n}, n \geq 2$. Since 


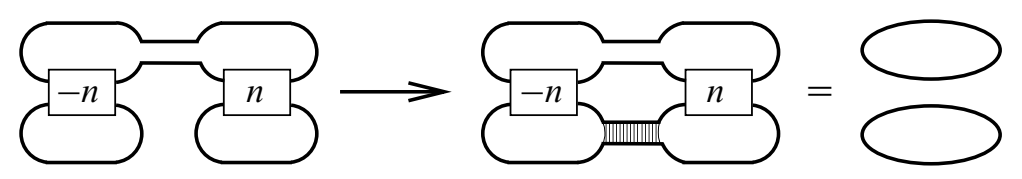

Figure 3: Ribbon move on $K(n, 1) \# K(n, n-1)$

the 2-fold cover of $S^{3}$ branched along any connected sum $K\left(p_{1}, q_{1}\right) \# K\left(p_{2}, q_{2}\right)$ is diffeomorphic to $L\left(p_{1}, q_{1}\right) \# L\left(p_{2}, q_{2}\right)$, by Lemma 3.6 this suffices to prove the proposition in Cases (3) and (5). A similar construction shows that if $p / q \in \mathcal{F}_{n}$ a connected sum $K(p, q) \# K(n, n-1)$ can be reduced to a 3-component unlink by 2 ribbon moves. Therefore the same connected sum bounds a ribbon surface $\Sigma$ with $\chi(\Sigma)=3-2=1$, and applying Lemma 3.6 as before proves the proposition in Case (4).

\section{Algebraic interlude}

In this section we recall some definitions and results from [7] and we establish some new results having a similar flavor. This material will be used in Section 5.

\section{Recollection of previous results}

Let $\mathbf{D}$ denote the intersection lattice $(\mathbb{Z},(-1))$, and $\mathbf{D}^{n}$ the orthogonal direct sum of $n$ copies of $\mathbf{D}$. Elements of $\mathbf{D}^{n}$ will also be called vectors. Fix generators $e_{1}, \ldots, e_{n} \in \mathbf{D}^{n}$ such that

$$
e_{i} \cdot e_{j}=-\delta_{i j}, \quad i, j=1, \ldots, n .
$$

Given a subset $S=\left\{v_{1}, \ldots, v_{n}\right\} \subseteq \mathbf{D}^{n}$, define

$$
\begin{array}{cl}
E_{i}^{S}:=\left\{j \in\{1, \ldots, n\} \mid v_{j} \cdot e_{i} \neq 0\right\}, & i=1, \ldots, n, \\
V_{i}:=\left\{j \in\{1, \ldots, n\} \mid e_{j} \cdot v_{i} \neq 0\right\}, & i=1, \ldots, n,
\end{array}
$$

and

$$
p_{i}(S):=\left|\left\{j \in\{1, \ldots, n\}|| E_{j}^{S} \mid=i\right\}\right|, \quad i=1, \ldots, n .
$$

Let $v_{1}, \ldots, v_{n} \in \mathbf{D}^{n}$ be elements such that, for $i, j \in\{1, \ldots, n\}$,

$$
v_{i} \cdot v_{j}= \begin{cases}-a_{i} \leq-2 & \text { if } i=j, \\ 0 \text { or } 1 & \text { if }|i-j|=1, \\ 0 & \text { if }|i-j|>1,\end{cases}
$$


for some integers $a_{i}, i=1, \ldots, n$.

Let $S=\left\{v_{1}, \ldots, v_{n}\right\} \subseteq \mathbf{D}^{n}$ be a subset which satisfies (4-1). Define the intersection graph of $S$ as the graph having as vertices $v_{1}, \ldots, v_{n}$, and an edge between $v_{i}$ and $v_{j}$ if and only if $v_{i} \cdot v_{j}=1$ for $i, j=1, \ldots, n$. The number of connected components of the intersection graph of $S$ will be denoted by $c(S)$. An element $v_{j} \in S$ is isolated, final or internal if the quantity

$$
\sum_{\substack{i=1 \\ i \neq j}}^{n}\left(v_{i} \cdot v_{j}\right)
$$

is equal to, respectively, 0,1 or 2 . In other words, $v_{j}$ is isolated or final if it is, respectively, an isolated vertex or a leaf of the intersection graph, and it is internal otherwise. Two elements $v, w \in \mathbf{D}^{n}$ are linked if there exists $e \in \mathbf{D}^{n}$ with $e \cdot e=-1$ such that

$$
v \cdot e \neq 0, \quad \text { and } \quad w \cdot e \neq 0 .
$$

A set $S \subseteq \mathbf{D}^{n}$ is irreducible if, given two elements $v, w \in S$, there exists a finite sequence of elements of $S$

$$
v_{0}=v, v_{1}, \ldots, v_{k}=w,
$$

such that $v_{i}$ and $v_{i+1}$ are linked for $i=0, \ldots, k-1$. A set which is not irreducible is reducible.

A subset $S=\left\{v_{1}, \ldots, v_{n}\right\} \subseteq \mathbf{D}^{n}$ is good if it is irreducible and its elements satisfy (4-1). If moreover $v_{i} \cdot v_{j}=1$ whenever $|i-j|=1$, we say that $S$ is standard.

Given a subset $S=\left\{v_{1} \ldots, v_{n}\right\} \subseteq \mathbf{D}^{n}$, define

$$
I(S):=\sum_{i=1}^{n}\left(-v_{i} \cdot v_{i}-3\right) \in \mathbb{Z} .
$$

Let Aut $\left(\mathbf{D}^{n}\right)$ be the group of automorphisms of $\mathbf{D}^{n}$ as an intersection lattice.

Lemma 4.1 [7, Lemma 2.4] Let $S=\left\{v_{1}, v_{2}, v_{3}\right\} \subseteq \mathbf{D}^{3}=\left\langle e_{1}, e_{2}, e_{3}\right\rangle$ be a good subset with $I(S)<0$. Then, up to applying to $S$ an element of $\operatorname{Aut}\left(\mathbf{D}^{3}\right)$ and possibly replacing $\left(v_{1}, v_{2}, v_{3}\right)$ with $\left(v_{3}, v_{2}, v_{1}\right)$, one of the following holds:

(1) $\left(v_{1}, v_{2}, v_{3}\right)=\left(e_{1}-e_{2}, e_{2}-e_{3},-e_{2}-e_{1}\right)$,

(2) $\left(v_{1}, v_{2}, v_{3}\right)=\left(e_{1}-e_{2}, e_{2}-e_{3}, e_{1}+e_{2}+e_{3}\right)$,

(3) $\left(v_{1}, v_{2}, v_{3}\right)=\left(e_{1}+e_{2}+e_{3},-e_{1}-e_{2}+e_{3}, e_{1}-e_{2}\right)$. 
Moreover,

$$
\left(p_{1}(S), p_{2}(S), c(S), I(S)\right)= \begin{cases}(1,1,1,-3) & \text { in case }(1) \\ (0,2,2,-2) & \text { in case }(2) \\ (0,1,2,-1) & \text { in case }(3)\end{cases}
$$

In particular, $\left(a_{1}, a_{2}, a_{3}\right) \in\{(2,2,2),(2,2,3),(3,3,2)\}$.

Lemma 4.2 [7, Lemma 2.6] Let $p>q \geq 1$ be coprime integers, and suppose that

$$
\frac{p}{q}=\left[a_{1}, \ldots, a_{n}\right]^{-}, \quad \frac{p}{p-q}=\left[b_{1}, \ldots, b_{m}\right]^{-},
$$

with $a_{1}, \ldots, a_{n} \geq 2$ and $b_{1}, \ldots, b_{m} \geq 2$. Then,

$$
\sum_{i=1}^{n}\left(a_{i}-3\right)+\sum_{j=1}^{m}\left(b_{j}-3\right)=-2 \text {. }
$$

Given elements $e, v \in \mathbf{D}^{n}$ with $e \cdot e=-1$, we denote by $\pi_{e}(v)$ the projection of $v$ in the direction orthogonal to $e$ :

$$
\pi_{e}(v):=v+(v \cdot e) e \in \mathbf{D}^{n}
$$

Definition 4.3 Let $S=\left\{v_{1}, \ldots, v_{n}\right\} \subseteq \mathbf{D}^{n}$ be a subset satisfying (4-1) and such that $\left|v_{i} \cdot e_{j}\right| \leq 1$ for every $i, j=1, \ldots, n$. Suppose that there exist $1 \leq h, s, t \leq n$ such that

$$
E_{h}^{S}=\{s, t\} \text { and } a_{t}>2 .
$$

Then, we say that the subset $S^{\prime} \subseteq\left\langle e_{1}, \ldots, e_{h-1}, e_{h+1}, \ldots, e_{n}\right\rangle \cong \mathbf{D}^{n-1}$ defined by

$$
S^{\prime}:=S \backslash\left\{v_{s}, v_{t}\right\} \cup\left\{\pi_{e_{h}}\left(v_{t}\right)\right\}
$$

is obtained from $S$ by a contraction, and we write $S \searrow S^{\prime}$. We also say that $S$ is obtained from $S^{\prime}$ by an expansion, and we write $S^{\prime} \nearrow S$.

Definition 4.4 Let $S^{\prime}=\left\{v_{1}, \ldots, v_{n}\right\} \subseteq \mathbf{D}^{n}, n \geq 3$, be a good subset, and suppose there exists $1<s<n$ such that $C^{\prime}=\left\{v_{s-1}, v_{s}, v_{s+1}\right\} \subseteq S^{\prime}$ is a connected component of the intersection graph of $S^{\prime}$, with $v_{s-1} \cdot v_{s-1}=v_{s+1} \cdot v_{s+1}=-2, v_{s} \cdot v_{s}<-2$ and $E_{j}^{S^{\prime}}=\{s-1, s, s+1\}$ for some $j$. Let $S \subseteq \mathbf{D}^{m}$ be a subset of cardinality $m \geq n$ obtained from $S^{\prime}$ by a sequence of expansions by final $(-2)$-vectors attached to $C^{\prime}$, so that $c(S)=c\left(S^{\prime}\right)$ and there is a natural one-to-one correspondence between the sets of connected components of the intersection graphs of $S$ and $S^{\prime}$. Then, the connected component $C \subseteq S$ corresponding to $C^{\prime} \subseteq S^{\prime}$ is a bad component of $S$. The number of bad components of $S$ will be denoted by $b(S)$.

Algebraic 83 Geometric Topology, Volume 7 (2007) 
In the arguments of the next two sections we shall need the following Proposition 4.5 and Lemmas 4.6 and 4.7.

Proposition 4.5 [7, Corollary 5.4] Suppose $n \geq 3$, and let $S_{n}=\left\{v_{1}, \ldots, v_{n}\right\} \subseteq \mathbf{D}^{n}$ be a good subset with no bad components and such that $I\left(S_{n}\right)<0$. Then $I\left(S_{n}\right) \in$ $\{-1,-2,-3\}$, there exists a sequence of contractions

$$
S_{n} \searrow S_{n-1} \searrow \cdots \searrow S_{3}
$$

such that, for each $k=3, \ldots, n-1$ the set $S_{k}$ is good, has no bad components and we have either

$$
\left(I\left(S_{k}\right), c\left(S_{k}\right)\right)=\left(I\left(S_{k+1}\right), c\left(S_{k+1}\right)\right)
$$

or

$$
I\left(S_{k}\right) \leq I\left(S_{k+1}\right)-1 \quad \text { and } \quad c\left(S_{k}\right) \leq c\left(S_{k+1}\right)+1 .
$$

Moreover:

(1) If $p_{1}\left(S_{n}\right)>0$ then $I\left(S_{n}\right)=-3, S_{n}$ is standard and one can choose the above sequence so that $I\left(S_{k}\right)=-3$ and $S_{k}$ is standard for every $k=3, \ldots, n-1$.

(2) If $I\left(S_{n}\right)+c\left(S_{n}\right) \leq 0$ then $S_{3}$ is given, up to applying an automorphism of $\mathbf{D}^{3}$, by either (1) or (2) in Lemma 4.1; if $I\left(S_{n}\right)+c\left(S_{n}\right)<0$ then the former case occurs.

Lemma 4.6 [7, Lemma 6.3] Let $S_{3} \subset \mathbf{D}^{3}$ be a good subset with $I\left(S_{3}\right)=-3$ and $c\left(S_{3}\right)=1$. Suppose that $S_{3} \nearrow \cdots \nearrow S_{k}$ is a sequence of expansions such that, for each $h=3, \ldots, k, S_{h}$ is good, has no bad component and $\left(I\left(S_{h}\right), c\left(S_{h}\right)\right)=(-3,1)$. Then, it is not possible to expand $S_{k}$ by an isolated (-3)-vector.

Lemma 4.7 [7, Lemma 6.2] Let $S_{3} \nearrow \ldots \nearrow S_{n}$ be a sequence of expansions such that, for each $k=3, \ldots, n, S_{k}$ is good, has no bad component and $\left(I\left(S_{k}\right), c\left(S_{k}\right)\right)=$ $(-2,2)$. Then,

(1) $p_{1}\left(S_{n}\right)=0, p_{2}\left(S_{n}\right)=2$ and $p_{3}\left(S_{n}\right)=n-2$.

(2) If $E_{i}^{S_{n}}=\left\{t, t^{\prime}\right\}$ then $v_{t}$ and $v_{t^{\prime}}$ are not internal and exactly one of them has square -2 .

(3) If $v_{t} \in S_{n}$ is not internal then there exists $i \in V_{t}$ such that $\left|E_{i}^{S_{n}}\right|=2$. 


\section{New results}

Lemma 4.8 Let $S_{3} \nearrow \ldots \nearrow S_{n}$ be a sequence of expansions of good subsets without bad components such that $\left(I\left(S_{k}\right), c\left(S_{k}\right)\right)=(-2,2)$ for every $k=3, \ldots, n$. Then, it is not possible to expand $S_{n}$ by an isolated (-3)-vector.

Proof Let $S_{n}=\left\{\bar{v}_{1}, \ldots, \bar{v}_{n}\right\} \subset\left\langle e_{1}, \ldots, e_{n}\right\rangle \cong \mathbf{D}^{n}$, and suppose that

$$
\mathbf{D}^{n+1} \cong\left\langle e_{1}, \ldots, e_{n+1}\right\rangle \supset S_{n+1}=\left\{v_{1}, \ldots, v_{n+1}\right\} \searrow S_{n}
$$

is a contraction obtained by eliminating the isolated vector $v_{n+1} \in S_{n+1}$ of square -3 . Since $S_{n}$ is good, has no bad components and $I\left(S_{n}\right)<0$, by [7, Proposition 5.2] we have $\left|\bar{v}_{i} \cdot e_{j}\right| \leq 1$ for every $i, j=1, \ldots, n$. Moreover, we may assume without loss $v_{n+1}=e_{1}+e_{2}+e_{n+1}$, with $\left|E_{n+1}^{S_{n+1}}\right|=2$. Then, since

$$
\begin{aligned}
\left|E_{1}^{S_{n}}\right|+\left|E_{2}^{S_{n}}\right|+1=\left|E_{1}^{S_{n+1} \backslash\left\{v_{n+1}\right\}}\right|+ & \left|E_{2}^{S_{n+1} \backslash\left\{v_{n+1}\right\}}\right|+\left|E_{n+1}^{S_{n+1} \backslash\left\{v_{n+1}\right\}}\right| \\
& \equiv \sum_{i \neq n+1} v_{i} \cdot v_{n+1} \quad(\bmod 2)=0 \quad(\bmod 2),
\end{aligned}
$$

we see that $\left|E_{1}^{S_{n}}\right|+\left|E_{2}^{S_{n}}\right|$ is odd. Therefore, in view of Lemma 4.7(1) we may assume $\left|E_{1}^{S_{n}}\right|=2$ and $\left|E_{2}^{S_{n}}\right|=3$. We claim that this is impossible. In fact, observe that by Lemma 4.7(2) we have $E_{1}^{S_{n}}=\left\{t, t^{\prime}\right\}$, with $\bar{v}_{t}$ and $\bar{v}_{t^{\prime}}$ not internal and $\bar{v}_{t} \cdot \bar{v}_{t}=-2$, say. Moreover, it is easy to check that $\bar{v}_{t}$ is final and $v_{t} \cdot e_{n+1}=0$. Therefore, since $v_{n+1} \cdot v_{t}=0$ we have $v_{t}= \pm\left(e_{1}-e_{2}\right)$. Since $E_{2}^{S_{n}}=\{t, s, r\}$ for some $s, r$ and $\bar{v}_{t}$ is not internal, we can assume $\bar{v}_{t} \cdot \bar{v}_{s}=0$. This implies $e_{1} \cdot \bar{v}_{s} \neq 0$, therefore $\bar{v}_{s}=\bar{v}_{t^{\prime}}$. It follows that $e_{1} \cdot \bar{v}_{r}=0$, hence $\bar{v}_{r} \cdot \bar{v}_{t}=1$. We conclude $\bar{v}_{t} \cdot \bar{v}_{t^{\prime}}=0$, so $v_{t^{\prime}}= \pm\left(e_{1}+e_{2}\right)+\cdots$, which is incompatible with the fact that $v_{n+1}=e_{1}+e_{2}+e_{n+1}$ is isolated in $S_{n+1}$.

Lemma 4.9 Let $S \subseteq \mathbf{D}^{n}$ be a good subset with no bad components and $I(S)<0$. Then, $c(S) \leq 2$.

Proof If $n \leq 2$ the conclusion is obvious, so we assume $n \geq 3$. By contradiction, suppose $c(S) \geq 3$. In view of Proposition 4.5 we have $I(S) \in\{-1,-2,-3\}$ and there is a sequence of contractions

$$
S=: S_{n} \searrow S_{n-1} \searrow \cdots \searrow S_{3} \subseteq \mathbf{D}^{3},
$$

where for each $k=3, \ldots, n$ the subset $S_{k}$ is good, has no bad components and either (4-3) or (4-4) holds. In particular, $I\left(S_{3}\right) \leq I\left(S_{4}\right) \leq \cdots \leq I\left(S_{n}\right)$.

Algebraic 83 Geometric Topology, Volume 7 (2007) 
First case: $I\left(S_{n}\right)=-3$ Since by Lemma 4.1 we have $-3 \leq I\left(S_{3}\right)$, in this case $I\left(S_{k}\right)=-3$ for every $k$. Hence (4-3) holds for every $k$, implying $c\left(S_{3}\right)=c\left(S_{n}\right) \geq 3$, and contradicting Lemma 4.1.

Second case: $I\left(S_{n}\right)=-2$ If $I\left(S_{3}\right)=-2$ then arguing as in the previous paragraph one gets a contradiction, so we may assume $I\left(S_{3}\right)=-3$. In this case (4-3) must hold for all but at most one index $k$. This clearly implies $c\left(S_{3}\right) \geq c\left(S_{n}\right)-1 \geq 2$, which contradicts Lemma 4.1.

Third case: $I\left(S_{n}\right)=-1$ If $I\left(S_{3}\right)=-1$ one gets a contradiction as before. If $I\left(S_{3}\right)=-2$ then in Sequence (4-5) there would an expansion by an isolated (-3)vector, going against Lemma 4.8. If $I\left(S_{3}\right)=-3$ then, since $c\left(S_{n}\right) \geq 3$ and by Lemma $4.1 c\left(S_{3}\right)=1$, in Sequence (4-5) there would be two expansions by isolated (-3)vectors, one of which would contradict Lemma 4.6.

Proposition 4.10 Let $S \subseteq \mathbf{D}^{n}$ be a good subset such that $I(S)+b(S)<0$. Then, $c(S) \leq 2$.

Proof By definition of bad component, there is a "minimal" good subset $S^{\prime}$ associated to $S$ with $I\left(S^{\prime}\right)=I(S), b\left(S^{\prime}\right)=b(S)$ and $c\left(S^{\prime}\right)=c(S)$. The set $S^{\prime}$ is obtained from $S$ via contractions by final $(-2)$-vectors, and each bad component of $S^{\prime}$ is of the form $\left\{v_{s-1}, v_{s}, v_{s+1}\right\}$, with $v_{s-1} \cdot v_{s-1}=v_{s+1} \cdot v_{s+1}=-2, v_{s} \cdot v_{s}<-2$ and $E_{j}^{S^{\prime}}=\{s-1, s, s+1\}$ for some $j$ and $s$. Then, it is easy to check that if for each bad component of $S^{\prime}$ we apply the transformation

$$
S^{\prime} \mapsto S^{\prime} \backslash\left\{v_{s}, v_{s+1}\right\} \cup\left\{\pi_{e_{j}}\left(v_{s}\right)\right\},
$$

the resulting subset $S^{\prime \prime}$ is good, has no bad components and satisfies $c\left(S^{\prime \prime}\right)=c(S)$ and $I\left(S^{\prime \prime}\right)=I\left(S^{\prime}\right)+b\left(S^{\prime}\right)=I(S)+b(S)<0$. Then, by Lemma 4.9 we have $c\left(S^{\prime \prime}\right)=c(S) \leq 2$.

\section{Proof of Theorem 1.1: second half}

In this section we use the results of Section 4 to show that if an oriented 3-manifold $Y$ homeomorphic to a connected sum of lens spaces $L_{1} \# \cdots \# L_{h}$ smoothly bounds a rational homology 4-ball, then $Y$ is homeomorphic to a connected sum where each summand is (up to orientation) homeomorphic to one of the manifolds listed in Theorem 1.1(1)-(5). The reader is referred to Section 4 for the algebraic terminology.

To start with, we observe that if $h=1$ then it follows from [7, Theorem 1.2] that $Y=L(p, q)$ with $p / q \in \mathcal{R}$. Therefore in this case the statement of Theorem 1.1 is established, and from now on we assume $h>1$. 
Recall that the lens space $L(p, q)$ is orientation-preserving diffeomorphic to the oriented boundary of the 4-dimensional plumbing $P(p, q)$ given by the weighted graph of Figure 4 , where $p / q=\left[a_{1}, \ldots, a_{n}\right]^{-}$. It is easy to check that the intersection form

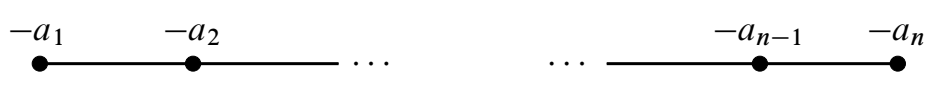

Figure 4: The weighted graph prescribing the plumbing $P(p, q)$

of the 4-dimensional plumbing $P(p, q)$ is negative definite. To simplify the notation, we shall also denote by $P_{L}$ the plumbing associated as above with a lens space $L$. Likewise, if $Y$ is a connected sum of lens spaces $L_{1} \# \ldots \# L_{h}$, we define $P_{Y}$ as the boundary connected sum

$$
P_{L_{1}} \downarrow \cdots \curvearrowleft P_{L_{h}} .
$$

Then, $-Y=\left(-L_{1}\right) \# \cdots \#\left(-L_{h}\right)$. Suppose that $Y=L_{1} \# \cdots \# L_{h}$ smoothly bounds a rational homology 4-ball $W_{Y}$. Consider the smooth, closed, negative 4-manifolds

$$
X_{Y}:=P_{Y} \cup_{\partial}\left(-W_{Y}\right), \quad X_{Y}^{\prime}:=P_{-Y} \cup_{\partial} W_{Y} .
$$

By Donaldson's theorem on the intersection form of definite 4-manifolds [3], the intersection forms of $X_{Y}$ and $X_{Y}^{\prime}$ are both standard diagonal.

Suppose that the intersection lattice of $X_{Y}$ is isomorphic to $\mathbf{D}^{n}$ (as defined in Section 4) and the intersection lattice of $X_{Y}^{\prime}$ is isomorphic to $\mathbf{D}^{n^{\prime}}$. Clearly, the groups $H_{2}\left(P_{Y} ; \mathbb{Z}\right) \cong \mathbb{Z}^{n}$ and $H_{2}\left(P_{-Y} ; \mathbb{Z}\right) \cong \mathbb{Z}^{n^{\prime}}$ have integral bases which satisfy Equations (4-1). Therefore, via the embeddings $P_{Y} \subset X_{Y}$ and $P_{-Y} \subset X_{Y}^{\prime}$ we can view such bases as subsets $S=S_{1} \cup \cdots \cup S_{h} \subset \mathbf{D}^{n}$ and $S^{\prime}=S_{1}^{\prime} \cup \cdots \cup S_{h}^{\prime} \subset \mathbf{D}^{n^{\prime}}$, where $S_{i}$, respectively $S_{i}^{\prime}$, originates from a basis of the corresponding summand $P_{L_{i}}$ of $P_{Y}$, respectively $P_{-L_{i}}$ of $P_{-Y}$. Observe that $c(S)=c\left(S^{\prime}\right)=h$ and $c\left(S_{i}\right)=c\left(S_{i}^{\prime}\right)=1$ for $i=1, \ldots, h$.

Definition 5.1 Let $S=\left\{v_{1}, \ldots, v_{k}\right\} \subset \mathbf{D}^{n}$. The string of $S$ is $\left(a_{1}, \ldots, a_{k}\right)$, where $a_{i}=-v_{i} \cdot v_{i}$ for $i=1, \ldots, k$.

We now briefly recall Riemenschneider's point rule [12]. Let $p>q>0$ be coprime integers, and suppose

$$
\frac{p}{q}=\left[a_{1}, \ldots, a_{l}\right]^{-}, a_{i} \geq 2, \quad \frac{p}{p-q}=\left[b_{1}, \ldots, b_{m}\right]^{-}, b_{j} \geq 2 .
$$

Algebraic 83 Geometric Topology, Volume 7 (2007) 
Then, the coefficients $a_{1}, \ldots, a_{l}$ and $b_{1}, \ldots, b_{m}$ are related by a diagram of the form

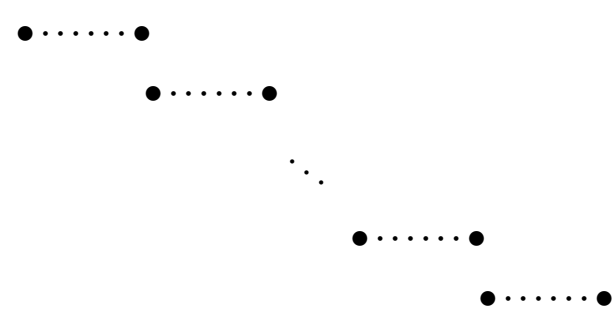

where the $i$-th row contains $a_{i}-1$ "points" for $i=1, \ldots, l$, and the first point of each row is vertically aligned with the last point of the previous row. The point rule says that there are $m$ columns, and the $j$-th column contains $b_{j}-1$ points for every $j=1, \ldots, m$. For example if $7 / 5=[2,2,3]^{-}$and $7 / 2=[4,2]^{-}$the corresponding diagram is given by

Lemma 5.2 We have $b(S)+b\left(S^{\prime}\right) \leq c(S)$.

Proof Suppose that $S_{i}$ is a bad component of $S$. Then, by Definition 4.4 the string of $S_{i}$ is obtained from the string $(2, a, 2), a \geq 3$, via a finite sequence of operations of type Lemma 3.3(1) or Lemma 3.3(2). Applying Riemenschneider's point rule (cf [7, Proof of Lemma 2.6]) one can easily check that, similarly, the string of $S_{i}^{\prime}$ is obtained from the string

$$
(3, \overbrace{2, \ldots, 2,3)}^{a-3}
$$

via a sequence of operations of type Lemma 3.3(1) or Lemma 3.3(2). Clearly, such a string is not the string of a bad component. This shows that, for each $i=1, \ldots, h$, at most one set among $S_{i}$ and $S_{i}^{\prime}$ can be a bad component, which implies the statement of the lemma.

Lemma 5.3 Up to replacing $S$ with $S^{\prime}$ we have $I(S)+c(S) \leq 0$ and $I(S)+b(S)<0$.

Proof We have $c(S)=c\left(S^{\prime}\right)$ and by Lemma 4.2 it follows that $I(S)+I\left(S^{\prime}\right)=$ $-2 c(S)$. Therefore, up to replacing $S$ with $S^{\prime}$ we may assume $I(S) \leq-c(S)$. If $I(S)<-c(S)$ then, since $b(S) \leq c(S)$, we have

$$
I(S)+b(S) \leq I(S)+c(S)<0
$$


and the lemma holds. Now assume $I(S)=-c(S)=I\left(S^{\prime}\right)$. By Lemma $5.2 b(S)+$ $b\left(S^{\prime}\right) \leq c(S)$. Therefore, up to replacing $S$ with $S^{\prime}$ we may assume $I(S)=-c(S)$ and $b(S)<c(S)$, so it follows that

$$
I(S)+b(S)<I(S)+c(S)=0 .
$$

This concludes the proof.

In view of Lemma 5.3, from now on we will assume:

$$
I(S)+c(S) \leq 0 \quad \text { and } \quad I(S)+b(S)<0 .
$$

Now there are two possibilities. Either $S$ is irreducible or it is reducible. We consider the two cases separately.

\section{First case: $S$ irreducible}

In this case $S$ is a good subset by definition. Since $I(S)+b(S)<0$, by Proposition 4.10, we have $h=c(S) \leq 2$. But we are assuming $h>1$, so we conclude $h=c(S)=2$. Since $b(S) \leq c(S)$, we must analyze the three subcases $b(S)=0, b(S)=1$ and $b(S)=2$.

First subcase: $b(S)=0 \quad$ By Proposition 4.5 there is a sequence of contractions of good subsets without bad components as in (4-2). Since $I\left(S_{3}\right) \leq I(S) \leq-c(S)=-2$, applying (4-3), (4-4) and Lemma 4.1 it is easy to check that $(I(S), c(S))=(-2,2)$ and either $\left(I\left(S_{3}\right), c\left(S_{3}\right)\right)=(-2,2)$ or $\left(I\left(S_{3}\right), c\left(S_{3}\right)\right)=(-3,1)$. The latter case is excluded by (4-3), (4-4) and Lemma 4.6. So we are left with the case $\left(I\left(S_{3}\right), c\left(S_{3}\right)\right)=$ $(-2,2)$. Since $I(S)=I\left(S_{3}\right)=-2$, by (4-3) and (4-4) Sequence (4-2) satisfies the assumptions of Lemma 4.7. Applying the lemma to $S_{k}$ for each $k=3, \ldots, n$ it is easy to check that the string associated with $S$ is a union of two strings $s_{1} \cup s_{2}$ related to each other by Riemenschneider's point rule. We conclude that, up to orientation, $Y=L_{1} \# L_{2}$ with $L_{1}=L(p, q)$ and $L_{2}=L(p, p-q)$ for some $p>q>0$, so Case (2) of Theorem 1.1 holds.

Second subcase: $b(S)=1$ By definition of bad component there is a sequence of contractions of good subsets

$$
S \searrow \cdots \searrow T
$$

obtained by erasing final (-2)-vectors, such that $c(T)=c(S)=2, b(T)=b(S)=1$, $I(T)=I(S) \leq-c(S)=-2$ and such that the bad component of $T$ is of the form $\left\{v_{s-1}, v_{s}, v_{s+1}\right\}$ with $v_{s-1} \cdot v_{s-1}=v_{s+1} \cdot v_{s+1}=-2$ and $v_{s} \cdot v_{s}<-2$. We can further reduce $T$ to a good subset without bad components $U$ as in the proof of Proposition 4.10, so that $I(U)=I(T)+1 \leq-1, c(U)=c(T)=2$ and one connected component 
of the intersection graph of $U$ consists of a single element. Moreover, by Proposition 4.5 there is a sequence of contractions

$$
U \searrow \cdots \searrow S_{3} \subset \mathbf{D}^{3}
$$

satisfying (4-3) and (4-4). In particular, $I(U) \geq I\left(S_{3}\right) \geq-3$. The case $I(U)=$ $I\left(S_{3}\right)=-3$ is impossible by (4-3) and (4-4) because $c(U)=2$ while by Lemma 4.1 the equality $I\left(S_{3}\right)=-3$ implies $c\left(S_{3}\right)=1$.

If $I(U)=-2$, the same analysis made in the first subcase above implies $\left(I\left(S_{3}\right), c\left(S_{3}\right)\right)$ $=(-2,2)$ and the string of $U$ must be of the form

$$
(n) \cup(\overbrace{2, \cdots, 2}^{n-1}), n \geq 2,
$$

therefore the string of $T$ is of the form

$$
(2, n+1,2) \cup(\overbrace{2, \cdots, 2}^{n-1}), \quad n \geq 2 .
$$

We conclude that the string of $S$ is of the form

$$
s_{n} \cup \overbrace{2, \cdots, 2}^{n-1}), \quad n \geq 2,
$$

where the string $s_{n}$ is obtained from $(2, n+1,2)$ by a finite sequence of operations as in Lemma 3.3. Applying Lemma 3.3 we conclude that, up to orientation, $Y=L_{1} \# L_{2}$ with $L_{1}=L\left(m^{2} n, m n k+1\right), L_{2}=L(n, n-1)$ for some $n \geq 2, m>k>0$ and $(m, k)=1$. In other words, Case (4) of Theorem 1.1 holds.

If $I(U)=-1$ then $I(S)=I(T)=I(U)-1=-2$. By Lemma 4.2 the subset $S^{\prime} \subset \mathbf{D}^{n^{\prime}}$ originating from the integral basis of $H_{2}\left(P_{-Y} ; \mathbb{Z}\right)$ satisfies $\left(I\left(S^{\prime}\right), c\left(S^{\prime}\right)\right)=(-2,2)$ and by Lemma 5.2 we have $b\left(S^{\prime}\right) \leq 1$. If $b\left(S^{\prime}\right)=0$, the argument given in the first subscase shows that $Y=L(p, q) \# L(p, p-q)$, so Case (2) of Theorem 1.1 holds. If $b\left(S^{\prime}\right)=1$, the same argument just used for $S$ shows that there is a sequence of contractions of good subsets

$$
S^{\prime} \searrow \cdots \searrow T^{\prime}
$$

obtained by erasing final $(-2)$-vectors, such that $c\left(T^{\prime}\right)=c\left(S^{\prime}\right)=2, b\left(T^{\prime}\right)=b\left(S^{\prime}\right)=$ $1, I\left(T^{\prime}\right)=I\left(S^{\prime}\right)=-2$. By Riemenschneider's point rule, the string of the bad component of $T^{\prime}$ is dual to the string of the non-bad component of $T$, and vice-versa. This immediately implies that the intersection graph of $S$ is a union of connected components $S_{1} \cup S_{2}$, where (i) the string of $S_{1}$ is obtained from $(2, a, 2)$, for some $a \geq 3$, by a finite sequence of operations as in Lemma 3.3 and (ii) the string of $S_{2}$ is the Riemenschneider dual of a string obtained in a similar way from $(2, b, 2)$, for 
some $b \geq 3$. Clearly $I\left(S_{1}\right)=a-5$ and by Lemma 4.2 we have $I\left(S_{2}\right)=-b+3$. But $I(S)=I\left(S_{1}\right)+I\left(S_{2}\right)=-2$ implies $a=b$, therefore applying Lemma 3.3 we conclude that, up to orientation, $Y=L_{1} \# L_{2}$ with $L_{1},-L_{2} \in \mathcal{F}_{a-1}$. Hence, Case (5) of Theorem 1.1 holds.

Third subcase: $b(S)=2$ As in the previous subcase, there is a sequence of contractions of good subsets by final $(-2)-$ vectors

$$
S \searrow \cdots \searrow T
$$

such that $c(T)=c(S)=2, b(T)=2$ and $I(T)=I(S)$. Erasing final (-2)-vectors as before we get a good subset $U \subseteq \mathbf{D}^{2}$, and it is easy to check that the string of $U$ must be equal to (2) $\cup(2)$. Therefore the string of $T$ is

$$
(2,3,2) \cup(2,3,2)
$$

and the string of $S$ is of the form $s_{n} \cup t_{n}$, where each one of $s_{n}$ and $t_{n}$ is obtained from $(2,3,2)$ by a finite sequence of operations as in Lemma 3.3. Applying Lemma 3.3 as in the previous subcase we see that, up to orientation, $Y=L_{1} \# L_{2}$ with $L_{1}=L\left(2 m^{2}, 2 m k+1\right)$ and $L_{2}=L\left(2 p^{2}, 2 p h+1\right)$, for some $m>k>0,(m, k)=1$ and $p>h>0,(p, h)=1$. Therefore Case (3) of Theorem 1.1 holds. Summarizing, so far we have proved:

Lemma 5.4 If $h \geq 2$ and the subset $S$ is irreducible, then $h=2$ and $Y$ is (possibly after reversing its orientation) homeomorphic to one of the manifolds given in Theorem 1.1(2)-(5).

\section{Second case: $S$ reducible}

In this case the set $S$ can be written as a disjoint union $S=\cup_{j} T_{j}$ of maximal irreducible subsets $T_{j} \subset S$. As observed in [7, Remark 2.1], the elements of $S$ are linearly independent over $\mathbb{Z}$ because they satisfy Equation (4-1). We claim that, for each index $j$,

$$
\left|T_{j}\right|=\left|\cup_{v_{i} \in T_{j}} V_{i}\right| .
$$

In fact, by the linear independence we have

$$
\left|\cup_{v_{i} \in T_{j}} V_{i}\right| \geq\left|T_{j}\right|,
$$

while by the maximality of each $T_{j}$ the union $\bigcup_{j}\left(\cup_{v_{i} \in T_{j}} V_{i}\right)$ is disjoint. Hence

$$
\left|\bigcup_{j}\left(\cup_{v_{i} \in T_{j}} V_{i}\right)\right|=\sum_{j}\left|\cup_{v_{i} \in T_{j}} V_{i}\right| .
$$

Algebraic ${ }^{3} \mathcal{G}$ Geometric Topology, Volume 7 (2007) 
So we have

$$
\sum_{j}\left|\bigcup_{v_{i} \in T_{j}} V_{i}\right| \geq \sum_{j}\left|T_{j}\right|=|S| \geq\left|\bigcup_{j}\left(\cup_{v_{i} \in T_{j}} V_{i}\right)\right|=\sum_{j}\left|\bigcup_{v_{i} \in T_{j}} V_{i}\right|,
$$

and the claim follows. Next, we claim that there exists at least one index $i$ such that $I\left(T_{i}\right)+c\left(T_{i}\right) \leq 0$ and $I\left(T_{i}\right)+b\left(T_{i}\right)<0$. Since $b\left(T_{j}\right) \leq c\left(T_{j}\right)$ for every $j$, it suffices to show that either (i) there is an index $i$ such that $I\left(T_{i}\right)+c\left(T_{i}\right)<0$ or (ii) there is an index $i$ such that $I\left(T_{i}\right)+c\left(T_{i}\right)=0$ and $I\left(T_{i}\right)+b\left(T_{i}\right)<0$. Since

$$
\sum_{j}\left(I\left(T_{j}\right)+c\left(T_{j}\right)\right)=I(S)+c(S) \leq 0
$$

if (i) does not hold then $I\left(T_{j}\right)+c\left(T_{j}\right)=0$ for every $j$. In this case, since we also have

$$
\sum_{j}\left(I\left(T_{j}\right)+b\left(T_{j}\right)\right)=I(S)+b(S)<0,
$$

we conclude that $I\left(T_{i}\right)+b\left(T_{i}\right)<0$ for some $i$, that is, that (ii) holds. By (5-3) and the last claim, we may apply Lemma 5.4 to $T_{i}$, viewed as a subset of the span of the $e_{k}$ 's hit by the vectors of $T_{i}$. Therefore, after possibly renaming the $L_{i}$ 's we conclude that either $L_{h}$ or $L_{h-1} \# L_{h}$ smoothly bounds a rational homology ball $Z$. Let $p \in \partial Z$, let $B \subseteq \partial Z$ a regular neighborhood of $p$, and let $W$ be a smooth rational homology ball with boundary $L_{1} \# \cdots \# L_{h}$. Clearly, the 3-manifold with boundary $\partial Z \backslash B$ can be viewed as a subset of $\partial W$ as well as $\partial Z$. Then, the space

$$
W \cup_{(\partial Z) \backslash B}(-Z)
$$

obtained by gluing $W$ and $-Z$ together along $\partial Z \backslash B$, is (after smoothing corners) a smooth rational homology ball with boundary $L_{1} \# \ldots \# L_{h^{\prime}}$, where $h^{\prime}$ is equal to $h-1$ or $h-2$, respectively. Thus, we have proved:

Lemma 5.5 If $h \geq 2$ and $S$ is reducible, after possibly renaming the $L_{i}$ 's one of the following holds:

- $L_{1} \# \cdots \# L_{h-1}$ and $L_{h}$ smoothly bound a rational homology ball.

- $L_{1} \# \cdots \# L_{h-2}$ and $L_{h-1} \# L_{h}$ smoothly bound a rational homology ball.

At the beginning of this section we observed that when $h=1$ the statement of Theorem 1.1 was proved in [7]. Therefore, the second half of Theorem 1.1 follows combining Lemmas 5.4 and 5.5. 


\section{References}

[1] G Burde, H Zieschang, Knots, de Gruyter Studies in Mathematics 5, Walter de Gruyter \& Co., Berlin (1985) MR808776

[2] A J Casson, J L Harer, Some homology lens spaces which bound rational homology balls, Pacific J. Math. 96 (1981) 23-36 MR634760

[3] S K Donaldson, The orientation of Yang-Mills moduli spaces and 4-manifold topology, J. Differential Geom. 26 (1987) 397-428 MR910015

[4] E Grigsby, D Ruberman, S Strle, Knot concordance and Heegaard Floer homology invariants in branched covers arXiv:math.GT/0701460

[5] S Jabuka, S Naik, Order in the concordance group and Heegaard Floer homology, Geom. Topol. 11 (2007) 979-994 MR2326940

[6] R Kirby, Problems in low-dimensional topology, from: "Geometric topology (Athens, GA, 1993)”, AMS/IP Stud. Adv. Math. 2, Amer. Math. Soc. (1997) 35-473 MR1470751

[7] P Lisca, Lens spaces, rational balls and the ribbon conjecture, Geom. Topol. 11 (2007) 429-472 MR2302495

[8] C Livingston, A survey of classical knot concordance, from: "Handbook of knot theory", Elsevier BV, Amsterdam (2005) 319-347 MR2179265

[9] C Manolescu, B Owens, A concordance invariant from the Floer homology of double branched covers arXiv:math.GT/0508065

[10] P Orlik, P Wagreich, Algebraic surfaces with $k^{*}$-action, Acta Math. 138 (1977) 43-81 MR0460342

[11] P Popescu-Pampu, The geometry of continued fractions and the topology of surface singularities, from: "Singularities in geometry and topology 2004", Adv. Stud. Pure Math. 46, Math. Soc. Japan, Tokyo (2007) 119-195 MR2342890

[12] O Riemenschneider, Deformationen von Quotientensingularitäten (nach zyklischen Gruppen), Math. Ann. 209 (1974) 211-248 MR0367276

[13] L Siebenmann, Exercises sur les noeuds rationnels, mimeographed notes, Orsay (1975)

Dipartimento di Matematica “L. Tonelli”, Largo Bruno Pontecorvo, 5, Università di Pisa, I-56127 Pisa, ITALY

lisca@dm.unipi.it

Received: 18 May 2007 Revised: 24 November 2007 